\title{
Dicer and Drosha predict outcomes in ovarian cancer
}

Messenger RNA (mRNA) levels of the RNase III enzymes, Dicer and Drosha, are associated with clinical outcomes in patients with ovarian cancer, according to US researchers. High expression of both of these enzymes was associated with increased survival, and low expression of Dicer was associated with poor clinical outcome.

RNA interference is currently being tested preclinically as a potential treatment for cancer. MicroRNA and small interfering RNA are used to regulate gene expression through RNA; Drosha cuts double-stranded RNA segments into short double-stranded RNA precursors in the nucleus. When these precursors move to the cytoplasm, Dicer slices them into mature double-stranded RNA segments. MicroRNAs are thought to have a role in cancer progression, and previous studies have reported decreased expression of microRNAs in ovarian cancer. However, the clinical effects of this decrease were unknown. These findings led William Meritt et al. to investigate whether amounts of Dicer and Drosha mRNA were linked to outcomes in patients with ovarian cancer.

\section{Low levels of Dicer were significantly associated with advanced tumor stage 77}

Using a quantitative reverse-transcription polymerase chain reaction assay the researchers measured the levels of Dicer and Drosha mRNA from 111 epithelial ovarian cancer samples obtained from the MD Anderson Cancer Center and the Brigham and Women's Gynecologic Oncology Tumor Bank, and compared them with expression in benign tumors.

Among the cancer specimens, $60 \%$ had decreased Dicer mRNA and 51\% had decreased Drosha mRNA; decreased levels of both mRNAs occurred in 39\% of specimens. Low levels of Dicer were significantly associated with advanced tumor stage. Low levels of Dicer and Drosha were significantly associated with reduced median overall survival, and tumors expressing high levels of both were associated with increased median survival. Decreased Drosha mRNA was indicative of a poor prognosis. The association of a poor outcome in those with reduced levels of these mRNAs was validated in independent clinical samples.

Overall this study shows that levels of Dicer and Drosha mRNA are variable in ovarian cancer specimens, and are clinically relevant as they are significantly associated with survival.

Mandy Aujla

Original article Merritt, W. M. et al. Dicer, Drosha and outcomes in patients with ovarian cancer. N. Engl. J. Med. 359, 2641-2650 (2008). 